\title{
National surveillance for non-polio enteroviruses in Canada: Why is it important?
}

\author{
Booth $\mathrm{TF}^{1,2 *}$, Grudeski $\mathrm{E}^{1}$, McDermid $\mathrm{A}^{1,2}$ \\ ${ }^{1}$ National Microbiology Laboratory, Public Health Agency of Canada, Winnipeg, MB \\ ${ }^{2}$ Department of Medical Microbiology, University of Manitoba, Winnipeg, MB \\ *Corresponding author: Tim.booth@phac-aspc.gc.ca
}

\begin{abstract}
A widespread outbreak of enterovirus D68 (EV-D68) was detected in association with respiratory illness in children across Canada and the United States during the autumn of 2014. The majority of cases were mild, but some were associated with more severe illness requiring hospitalization; some of the cases also had neurological symptoms including paralysis, and three deaths were reported in British Columbia. EV-D68 is one of many enteroviruses that include Coxsackieviruses, echovirusesand polio virus. Other than polio virus, there are no vaccines available for the prevention of enterovirus infections, nor are there any antiviral medications that have been approved for their treatment. More than 46 different serotypes have been identified to be circulating in Canada over the last 25 years. Until 2014, EV-D68 was rare. Routine genotyping surveillance done by Canada's National Microbiology Laboratory (NML) identified only 85 isolates of EV-D68 between 1991 and 2013, while 282 were detected between July and October 2014. The complexity of the epidemiology of these enteroviruses demonstrates the need for genotype surveillance, to detect outbreaks spatially and temporally, to determine their relative incidence and impact on the population, and to investigate evolutionary trends, such as recombination events, that are thought to play an important part in strain variation and emergence of epidemic strains. In particular, it is important to carry out virological testing on unusual cases of paralysis in children, and to genotype and sequence any viruses identified. Submission of specimens (virus cultures, stool, cerebrospinal fluid or respiratory specimens) from any such cases to the National Centre for Enteroviruses at NML is encouraged.
\end{abstract}

\section{Introduction}

Enterovirus D68 (EV-D68) captured public attention between August and October of 2014 when a widespread outbreak was detected in association with respiratory illness in children across Canada and the United States (1-6). The majority of cases were mild and involved influenza-like symptoms, such as fever, cough, rhinitis, pharyngitis, bronchitis and myalgia. A proportion of these cases of EV-D68 infection were associated with more severe illness requiring hospitalization. The hospitalized cases involved pneumonia, bronchiolitis and respiratory distress, and underlying asthma appeared to be the main risk factor. Some of the cases also had neurological symptoms including paralysis, and three deaths were reported in British Columbia (6). In the United States, the Centers for Disease Control and Prevention (CDC) tested about 2,600 specimens for EV-D68 during the late summer of 2014 and found that about $36 \%$ of them were positive for the virus, including 12 patients who died, and the infection was confirmed in 1,152 patients in 49 states (7). In Canada, the National Microbiology Laboratory (NML) tested 970 specimens for EV-D68 and identified 282 positive cases between August and October of 2014. It has been suggested that the EV-D68 outbreak in the United States could be related to an increase in cases of acute flaccid myelitis, an unexplained neurological illness involving limb weakness in children that coincided with the increased detection of EV-D68 (8). The purpose of this article is to provide an overview of the complexity of the aetiology and epidemiology of non-polio enterovirus diseases and to identify why it is important to track this group of infections. There is a need to carry out further laboratory-based epidemiological surveillance for possible links between enterovirus infection and emerging paralytic illnesses.

Enteroviruses belong to the picornavirus family, a large and diverse group of small ribonucleic acid (RNA) viruses characterized by a single positive-strand genomic RNA, and include four major species, A, B, C and D, of which polio virus strains are members of the $C$ group (9). Poliovirus thus shares many biochemical and physical 
characteristics with other human enteroviruses (which include Coxsackieviruses, echoviruses, and rhinoviruses: the latter are common cold viruses). Non-polio enteroviruses cause many clinical syndromes, notably hand-footand-mouth disease, aseptic meningitis, flaccid paralysis, myocarditis, pneumonia and respiratory disease, fevers, gastroenteritis, hepatitis, and pancreatitis. Most of these infections affect children disproportionately, and many are potentially fatal. It is also likely that enterovirus infections are highly under-diagnosed, since laboratory testing is often not carried out. Other than polio virus, there are no vaccines available for the prevention of enterovirus infections, nor are there any antiviral medications that have been approved for their treatment. In Canada, as in many temperate regions, enterovirus infections peak in the late summer and early autumn months (Figure 1). Enteroviruses are usually transmitted by a faecal-oral route, through contaminated water supplies, and by close contact with infected persons. Enteroviruses can be detected in stool specimens, respiratory specimens, and are also found in cerebrospinal fluid specimens of infected persons (10). Many enteroviruses including EV-D68 are also shed in respiratory secretions suggesting that a respiratory route of infection is also involved in the transmission of EV-D68. So far there are no reports of finding EV-D68 in the nervous system which could provide a hypothetical mechanism to explain the possibility of neuropathogenic effects including paralysis.

Figure 1: The seasonality of echovirus infections in Canada, from 1990 to $2009^{a}$

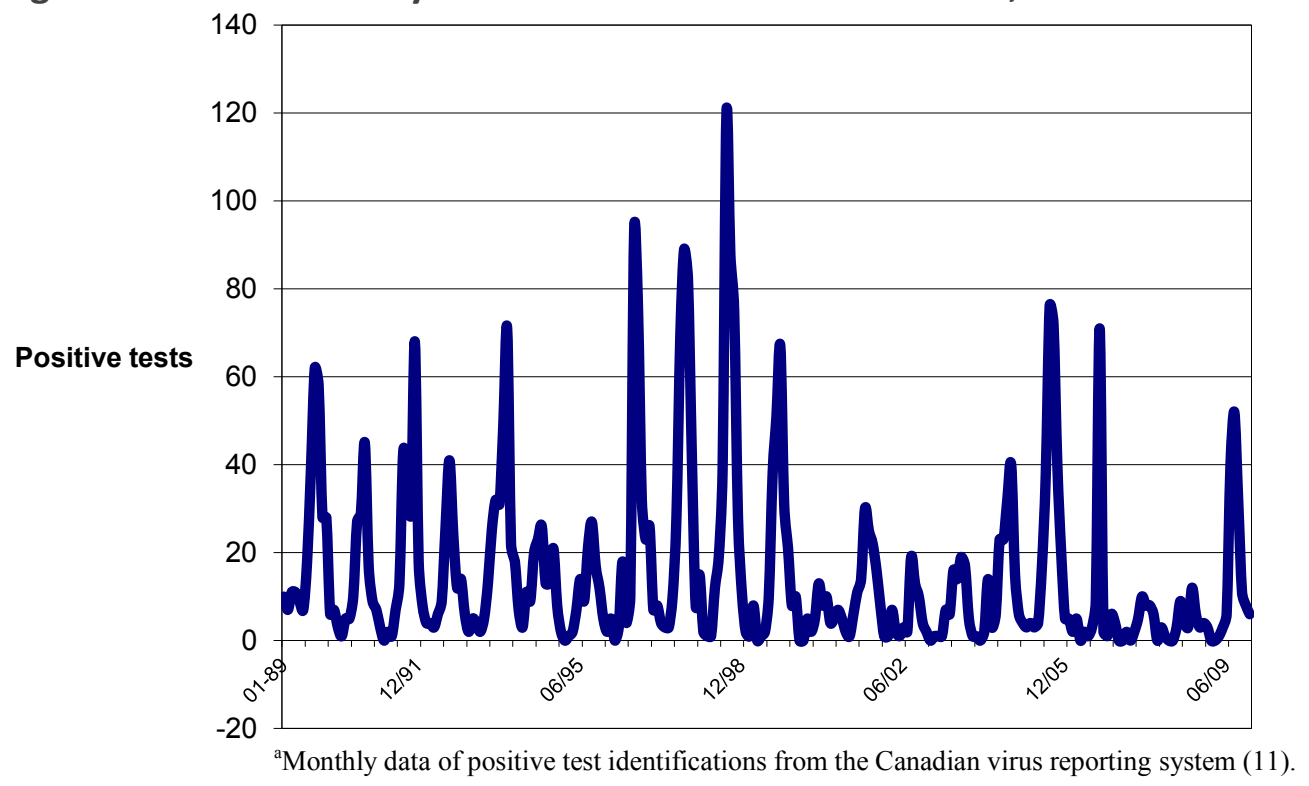

\section{Enterovirus surveillance in Canada and the emergence of EV-D68}

EV-D68 was rarely identified in Canada between1990-2014. Routine genotyping surveillance done by the National Centre for Enteroviruses at the Public Health Agency of Canada's National Microbiology Laboratory identified only 85 isolates of EV-D68, Then there were 282 positive cases out of 970 tested occurred between July and October of 2014 (Figure 2). EV-D68 is usually associated with respiratory infections and mild cold-like illnesses, but there are reports that it has occasionally been associated with more acute respiratory diseases in children, and rarely in association with central nervous system disease (12). Outbreaks of EV-D68 infection in children were detected in 2009 and 2010 in many parts of the world (12-19). In Canada, there is evidence from our laboratory genotyping surveillance that minor outbreaks of EV-D68 also occurred during 2009 (15 cases) and 2010 (23 cases) (Figure 2). 
Figure 2: Numbers of cases of EV-68 infection identified in Canada by molecular genotyping at the National Microbiology Laboratory, 2004-2014

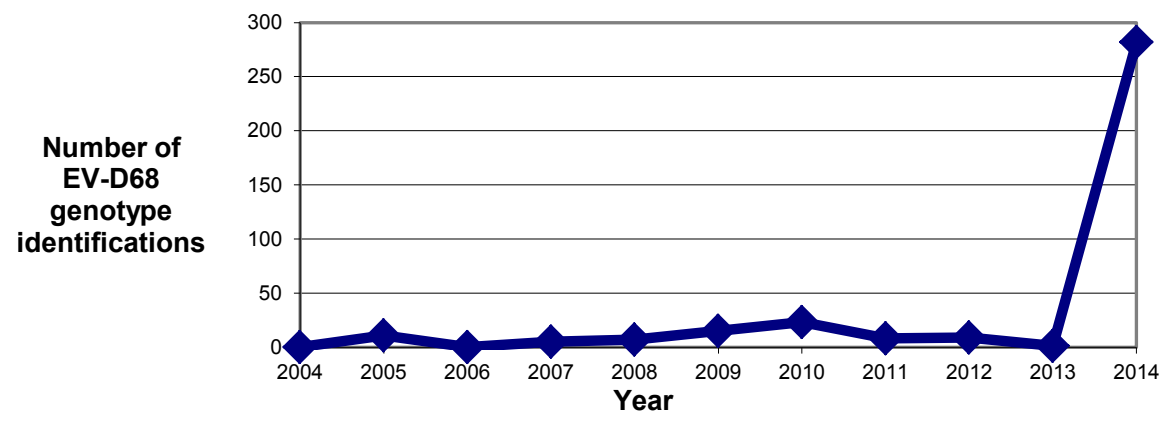

The NML routinely genotypes enterovirus specimens of as part of its diagnostic services; this testing also has the added benefit of providing surveillance data on the incidence of various enterovirus serotypes in Canada over the years, and an ability to quickly identify any unusual outbreaks. More than 46 different serotypes have been identified to be circulating frequently in Canada over the last 25 years. Data for the 20 most prevalent enteroviruses are shown in Table 1. A year-by-year analysis shows periodic peaks and lows for each serotype: echovirus 30 is the most common non-polio enterovirus circulating in Canada and there were major outbreaks in 1998, 2006 and 2009. Echovirus 7 caused an outbreak in 2002, and there was a peak of echovirus 5 in 2007. Coxsackievirus A16 (CV-A16) is the most common cause of hand-foot-and-mouth disease; and CV-A9, which is also commonly circulating in Canada and is associated with these disease outbreaks, had a major peak of laboratory identifications in 2003. 
Table 1: The number of virus isolations for the $\mathbf{2 0}$ most prevalent enterovirus genotypes in Canada from 1991 to 2013, in surveillance carried out by the National Centre for Enteroviruses, National Microbiology Laboratory

\begin{tabular}{|c|c|c|}
\hline Enterovirus serotype & Total isolations & $\%$ of total \\
\hline Echovirus 30 (E-30) & 408 & 15,5 \\
\hline Coxsackievirus A16 (CV-A16) & 196 & 7,5 \\
\hline Echovirus $11(\mathrm{E}-11)$ & 183 & 6,9 \\
\hline Coxsackievirus (CV-A9) & 177 & 6,7 \\
\hline Echovirus 18 (E-18) & 149 & $5,6 \%$ \\
\hline Echovirus 25 (E-25) & 137 & 5,2 \\
\hline Echovirus 9 (E-9) & 116 & 4,4 \\
\hline Coxsackievirus (CV-B4) & 111 & 4,2 \\
\hline Coxsackievirus (CV-B3) & 104 & 3,9 \\
\hline Enterovirus A71 (EV-A71) & 101 & 3,8 \\
\hline Coxsackievirus (CV-B2) & 98 & 3,7 \\
\hline Coxsackievirus (CV-B5) & 97 & 3,6 \\
\hline Echovirus $6(\mathrm{E}-6)$ & 97 & 3,6 \\
\hline Enterovirus D68 (EV-D68) & 85 & 3,2 \\
\hline Echovirus 7 (E-7) & 83 & 3,1 \\
\hline Coxsackievirus (CV-B1) & 80 & 3,0 \\
\hline Echovirus 5 (E-5) & 77 & 2,9 \\
\hline Echovirus 4 (E-4) & 58 & 2,2 \\
\hline Echovirus 22 & 45 & 1,7 \\
\hline Echovirus $3(\mathrm{E}-3)$ & 34 & 1,3 \\
\hline
\end{tabular}

\section{The case for surveillance}

Genotype surveillance is needed because of the complexity of the epidemiology of these enteroviruses, both in the wide variety of genotypes that are present in the population and in the diverse range of diseases that they are associated with. The aim is to detect outbreaks spatially and temporally and to determine their relative incidence and impact on the population. In addition, this surveillance gives warning of emerging infections such as EV-A71 and EV-D68, as well as the capability to follow genetic evolution in strains that could be associated with increased pathogenicity. In particular, it will be important to carry out virological testing on unusual cases of paralysis in children, and to genotype and sequence any viruses identified. We encourage submission of specimens from any such cases to the enterovirus section at NML. Ideally these specimens can be virus cultures, but cerebrospinal fluid, stool or respiratory specimens are also suitable. We also need to maintain vigilance for polio virus now that we are at a critical point in the global eradication of poliomyelitis. 


\section{Acute flaccid paralysis and non-polio enteroviruses}

Acute flaccid paralysis (AFP) is defined as sudden onset of reduced muscle tone and weakness without obvious cause (i.e., trauma) and is usually characterized by looseness and flexibility in limbs, and a lack of strength in moving and controlling the muscles. It may be caused by Guillain-Barré syndrome (an autoimmune disorder affecting the peripheral nervous system), and by a number of agents including poliovirus, some non-polio enteroviruses, echoviruses, adenovirus, West Nile virus, and campylobacter infections. Any part of the body can be affected — not only the limbs — and this may result in difficulty in breathing, suffocation and death. Thus, AFP has a very broad clinical syndrome, can have a wide range of causes, and can appear similar to other neurological syndromes, including, for example, transverse or anterior myelitis and traumatic neuritis (20).

It is well known that non-polio enteroviruses, such as Coxsackieviruses, echoviruses, and many enteroviruses, including types $70,71,89,90,91,96,99,102$, and 114 , are associated with numerous neurological clinical manifestations, such as encephalitis, meningitis and paralytic disease, including AFP-like syndromes (21). Of these viruses, EV-A71 has been found to be the most commonly associated with non-polio flaccid paralysis (20) and with neurological disease, including fatal encephalomyelitis (22). EV-A71 emerged during the 1990s as an agent that causes periodic, extensive outbreaks of hand-foot-and-mouth disease in children in China and other parts of Asia (23), and the viral sub-genotypes involved in these outbreaks also spread throughout Southeast Asia and Australia. An outbreak of EV-A71 in Taiwan in 1998 caused 1.5 million infections and 78 deaths (23). A particularly large outbreak of EV-A71 occurred in China in 2009; although the total number of cases was not reported in all provinces, it was clear that a small proportion of these developed severe complications that included neurological symptoms and deaths (24). Nevertheless, severe cases of EV-A71, especially with neurological involvement, are relatively rare, and most children affected recover completely. Antivirals that are effective for this class of viruses are still at the development stage. Efforts are also underway to develop vaccines for the control of continuing EV-A71 outbreaks in Asia. In addition to hand-foot-and-mouth disease, non-polio enterovirus infections are also the likely cause of about $50 \%$ of all cases of aseptic meningitis (25). Thus, there is already a great deal of evidence that enterovirus infections can cause neurological diseases including paralysis.

A pilot study in Canada during September 2014 in seven provinces and territories collected epidemiological data for 268 hospitalized cases of EV-D68 infection and found only 3 neurological cases and no deaths (26). The rarity of these neurological cases means that much larger population studies will be needed to more fully investigate the possible link between EV-D68 and paralysis. One difficulty is that, other than polio, enterovirus infections are not nationally notifiable in Canada, and so the true number of laboratory-confirmed cases may not be reported. In addition, the true incidence of infection is likely to be much higher than the numbers of laboratory-confirmed cases, since the majority of infections never undergo virological testing. Therefore, it will be important to continue this enhanced surveillance during the next enterovirus season in 2015, to determine if EV-D68 returns and, if so, to measure its impact on the population, especially in children. More severe cases of non-polio enterovirus infection may also be identified through Canada's AFP surveillance system, or through the NML's national enterovirus genotype surveillance program. It is thus important to continue with these laboratory-based virological surveillance systems to be able to detect further emerging enterovirus disease, should it occur in Canada.

\section{Conclusion}

The identification of associations or causal links between enterovirus disease outbreaks and more serious illnesses such as paralysis have posed some challenges that can only be addressed by additional widespread surveillance. This should involve the collection of comprehensive clinical and epidemiological data in conjunction with laboratory testing that includes molecular typing and sequencing of the viruses. Although there is no proven link between EV-D68 and paralysis in children, enteroviruses are well known as agents that are associated with neurological illnesses and paralysis in a small proportion of infected cases. The emergence of an enterovirus, EVD68, that was hitherto rarely identified as a cause of illness, as an agent that is capable of causing widespread outbreaks of mild to severe respiratory disease, is a new health risk that warrants continued investigation and close monitoring. A next step is to increase the number of specimens submitted for enterovirus typing, and to improve the level of clinical epidemiological data included in these submissions. Ideal specimens for enterovirus testing are virus cultures, stool, cerebrospinal fluid or respiratory swabs or aspirates. Surveillance for non-polio enteroviruses helps to improve confidence in Canada's polio-free status, as well as identifying the incidence and 
impact of enteroviruses on health and for monitoring for emerging agents that pose a new risk to health such as EV-D68 and EV-A71 viruses.

\section{Acknowledgements}

The authors thank Rhiannon Huzarewich, Lily MacDonald, Michelle Gusdal and Russel Mandes for their excellent technical assistance. The support of Canadian provincial public health laboratories and hospital laboratories is also gratefully acknowledged.

\section{Funding}

The enterovirus program is funded by the Public Health Agency of Canada.

\section{Conflict of interest}

None.

\section{References}

(1) Midgley CM, Jackson MA, Selvarangan R, Turabelidze G, Obringer E, Johnson D, et al. Severe respiratory illness associated with enterovirus D68-Missouri and Illinois, 2014. MMWR. 2014;63(36):798-9.

(2) Hasegawa S, Hirano R, Okamoto-Nakagawa R, Ichiyama T, Shirabe K. Enterovirus 68 infection in children with asthma attacks: Virus-induced asthma in Japanese children. Allergy. 2011;66(12):1618-20. doi: 10.1111/j.13989995.2011.02725.x.

(3) Human enterovirus D68-North America (20): Update. ProMED. 2014 Dec 15. Archive No. 20141215.3035008. http://www.promedmail.org/direct.php?id=3035008

(4) Pastula DM, Aliabadi N, Haynes AK, et al. Acute neurologic illness of unknown etiology in children-Colorado, August-September 2014. MMWR. 2014;63(40):901-2. doi: mm6340a5 [pii].

(5) Fonseca K, Kellner JD, Talbot J, et al. Human enterovirus 68-Canada: (Alberta). ProMED. 2014 Sep 16 [cited 2015 Jan 6]. Archive No. 20140916.2775534. http://www.promedmail.org/direct.php?id=2775534

(6) BC Centre for Disease Control. British Columbia Influenza Surveillance Bulletin. Influenza Season 2014-15, Number 11, Weeks 51-52, December 14 to 27, 2014 [cited 2015 Jan 20]. http://www.bccdc.ca/NR/rdonlyres/B8298409-17F2-48B3-B146519D40AB5A06/0/InfluBulletin_Number11_Weeks5152_201415.pdf

(7) Centers for Disease Control and Prevention (CDC). Enterovirus D68 in the United States, 2014 [updated 2014 Oct 14 ; cited 2015 Jan 6]. http://www.cdc.gov/non-polio-enterovirus/outbreaks/EV-D68-outbreaks.html

(8) Roos R, Center for Infectious Disease Research and Policy (CIDRAP). CDC: Link between polio-like illness and EVD68 looks likely. CIDRAP News. 2014 Dec 2.

http://www.cidrap.umn.edu/news-perspective/2014/12/cdc-link-between-polio-illness-and-ev-d68-looks-likely

(9) Van Regenmortel MHV, et al., editors. International Union of Microbiological Societies, Virology Division. Virus taxonomy: Seventh report of the International Committee on Taxonomy of Viruses. San Diego: Academic Press; 2001.

(10) Jaramillo-Gutierrez G, Benschop KS, Claas EC, de Jong AS, van Loon AM, Pas SD, Pontesilli O, Rossen JW, Swanink CM, Thijsen S, van der Zanden AG, van der Avoort HG, Koopmans MP, Meijer A. September through October 2010 multi-centre study in the Netherlands examining laboratory ability to detect enterovirus 68, an emerging respiratory pathogen. J Virol Methods. 2013 Jun;190(1-2):53-62. doi: 10.1016/j.jviromet.2013.02.010. Epub 2013 Mar 1.

(11) Laboratory evidence of human viral and selected non-viral infections in Canada 1989 to 1996. CCDR. 1998 Oct;24S7. http://www.collectionscanada.gc.ca/webarchives/20071207052454/http://www.phacaspc.gc.ca/publicat/ccdr-rmtc/98pdf/24s7e.pdf

(12) Kaida A, Kubo H, Sekiguchi J, et al. Enterovirus 68 in children with acute respiratory tract infections, Osaka, Japan. Emerg Infect Dis. 2011;17(8):1494-7. doi: 10.3201/eid1708.110028.

(13) Ikeda T, Mizuta K, Abiko C, et al. Acute respiratory infections due to enterovirus 68 in Yamagata, Japan between 2005 and 2010. Microbiol Immunol. 2012;56(2):139-43. doi: 10.1111/j.1348-0421.2012.00411.x.

(14) Meijer A, Benschop KS, Donker GA, van der Avoort HG. Continued seasonal circulation of enterovirus D68 in the Netherlands, 2011-2014. Eurosurveillance. 2014 Oct 23;19(42). http://www.eurosurveillance.org/ViewArticle.aspx?Articleld=20935

(15) Meijer A, van der Sanden S, Snijders BE, et al. Emergence and epidemic occurrence of enterovirus 68 respiratory infections in the Netherlands in 2010. Virology. 2012;423(1):49-57. doi: 10.1016/j.virol.2011.11.021. 
(16) Jacobson LM, Redd JT, Schneider E, et al. Outbreak of lower respiratory tract illness associated with human enterovirus 68 among American Indian children. Pediatr Infect Dis J. 2012;31(3):309-12. doi:

10.1097/INF.0b013e3182443eaf.

(17) Centers for Disease Control and Prevention (CDC). Clusters of acute respiratory illness associated with human enterovirus 68-Asia, Europe, and United States, 2008-2010. MMWR. 2011;60(38):1301-4. doi: mm6038a1.

(18) Imamura T, Fuji N, Suzuki A, et al. Enterovirus 68 among children with severe acute respiratory infection, the Philippines. Emerg Infect Dis. 2011;17(8):1430-5. doi: 10.3201/eid1708.101328.

(19) Ayscue P, Van Haren K, Sheriff H, Waubant E, Waldron P, Yagi S, Yen C, Clayton A, Padilla T, Pan C, Reichel J, Harriman K, Watt J, Sejvar J, Nix WA, Feikin D, Glaser C. Acute flaccid paralysis with anterior myelitis-California, June 2012-June 2014. MMWR. 2014 Oct 10;63(40):903-6.

(20) Rao CD, Yergolkar P, Shankarappa KS. Antigenic diversity of enteroviruses associated with nonpolio acute flaccid paralysis, India, 2007-2009. Emerg Infect Dis. 2012 Nov; 18(11). doi: 10.3201/eid1811.111457. http://dx.doi.org/10.3201/eid1811.111457

(21) Rhoades RE, Tabor-Godwin JM, Tsueng G, Feuer R. Enterovirus infections of the central nervous system. Virology. 2011 Mar 15;411(2):288-305. doi: 10.1016/j.virol.2010.12.014. Epub 2011 Jan 20.

(22) Lum LC, Wong KT, Lam SK, Chua KB, Goh AY, Lim WL, Ong BB, Paul G, AbuBakar S, Lambert M. Fatal enterovirus 71 encephalomyelitis. J Pediatr. 1998 Dec;133(6):795-8.

(23) Wu W-H, Kuo T-C, Lin Y-T, Huang S-W, Liu H-F, et al. Molecular epidemiology of enterovirus 71 infection in the central region of Taiwan from 2002 to 2012. PLoS One. 2013 Dec 13;8(12):e83711. doi:10.1371/journal.pone.0083711.

http://journals.plos.org/plosone/article?id=10.1371/journal.pone.0083711

(24) BBC News. China on alert as virus spreads. 2008 May 3 [cited 2015 Jan 6]. http://news.bbc.co.uk/2/hi/asia-pacific/7381741.stm

(25) Michos AG, Syriopoulou VP, Hadjichristodoulou C, Daikos GL, Lagona E, Douridas P, Mostrou G, Theodoridou M. Aseptic meningitis in children: Analysis of 506 cases. PLoS One. 2007 Aug 1;2(7):e674.

(26) Edwin JJ, Reyes Domingo F, Booth TF, et al. Surveillance summary of hospitalized paediatric enterovirus D68 cases in Canada, September 2014. CCDR. 2015 Feb 20;41S1:2-8. 